\title{
Comparison of MR Imaging Findings between Extraligamentous and Subligamentous Disk Herniations in the Lumbar Spine
}

\author{
K.-J. Oh, J.W. Lee, B.L. Yun, S.T. Kwon, K.-W. Park, J.S. Yeom, and H.S. Kang
}

\begin{abstract}
BACKGROUND AND PURPOSE: The method of treating an HIVD in the lumbar spine may depend on the integrity of the PLL. The purpose of this study was to analyze and compare the MR imaging findings of extraligamentous and subligamentous HIVDs in the lumbar spine.
\end{abstract}

MATERIAL AND METHODS: One hundred seventeen patients ( $M / F=71: 46$; mean age, 47 years; age range, 15-79 years) underwent lumbar spine MR imaging and disk surgery (extraligamentous/subligamentous = 66:51) from May 2003 to November 2006. Two radiologists in consensus retrospectively reviewed all MR images, focusing on 10 criteria.

RESULTS: The following 5 criteria are suggestive of extraligamentous HIVD in the lumbar spine: 1) spinal canal compromised for more than half its dimension, 2) internal signal difference in the HIVD, 3) an ill-defined margin of the HIVD, 4) disruption of the continuous low-signalintensity line covering the HIVD, and 5) the presence of an internal dark line in the HIVD $(P<.05)$. When we combined these 5 MR imaging criteria, the sensitivity, specificity, accuracy, and odds ratio were $77.3 \%, 74.5 \%, 76.1 \%$, and $9.93(P<.0001)$.

CONCLUSIONS: Our proposed 5 MR imaging criteria will be helpful in differentiating extraligamentous and subligamentous HIVDs in the lumbar spine.

ABBREVIATIONS: $B M=$ bone marrow; HIVD = herniated intervertebral disk; NPV = negative predictive value; PLL = posterior longitudinal ligament; PPV = positive predictive value

$\mathbf{L}^{2}$

umbar HIVD is one of the most common causes of low back pain. To control symptoms produced by a lumbar HIVD, various treatments have been used for several years. Among these, some minimally invasive methods, such as percutaneous disk decompression and endoscopic diskectomy, can be adapted for contained disk herniation only because the procedures for noncontained disk herniations would have more chance of residual disk material in the epidural space or nerve root injury. Therefore, in considering these treatment options, the integrity of the PLL is crucial on preoperative MR imaging. ${ }^{1-4}$ However, to the best of our knowledge, there are only 2 reports on the integrity of the PLL in lumbar HIVD on MR images. ${ }^{5,6}$

During microscopic diskectomy from a posterior approach, the integrity of the PLL can be easily determined on the operative

Received April 21, 2012; accepted after revision June 12

From the Departments of Radiology (K.-J.O., J.W.L., B.L.Y., H.S.K.) and Orthopedic Surgery (K.-W.P., J.S.Y.), Seoul National University Bundang Hospital, Gyeongi-Do, South Korea; and Department of Radiology (K.-J.O., S.T.K.), Chungnam National University (Graduate School) College of Medicine, Daejeon, South Korea.

Please address correspondence to Joon Woo Lee, MD, Department of Radiology, Seoul National University Bundang Hospital, 300 Gumidong, Bundag-Gu, Seong Nam, Gyeongi-Do, 463-707 Korea, joonwoo2@gmail.com

http://dx.doi.org/10.3174/ajnr.A3258 field. In our institute, surgeons have described the integrity of PLL on surgical records after microdiskectomy as extraligamentous or subligamentous lumbar HIVD and have discussed these findings with radiologists during conferences. We thought that if we found clues for PLL integrity from surgically confirmed cases during microdiskectomy, those clues could provide a selection guideline for considering percutaneous disk intervention or endoscopic disk decompression.

The purpose of this study was to compare the MR imaging findings of extraligamentous lumbar HIVD with subligamentous lumbar HIVD on the basis of surgically confirmed cases.

\section{MATERIALS AND METHODS Patients}

According to the data base of the department of orthopedic surgery in our hospital, 150 patients underwent disk surgery after spine MR imaging from May 2003 to November 2006 at our institution. One radiologist retrospectively reviewed the medical records of those 150 patients, focusing on the surgical record, admission notes, and discharge summary. Among these cases, 23 were excluded due to the difficulty or ambiguity of the PLL evaluation and the HIVD location without PLL coverage. These included the following: 1) patients with prior lumbar disk surgery, 
2) an unclear description of the PLL integrity on the surgical record, and 3 ) foraminal or extraforaminal lumbar HIVD. We also excluded 10 cases due to delay of surgery of $>1$ month after spine MR imaging. Finally, 117 patients $(\mathrm{M} / \mathrm{F}=71: 46$; mean age, 47 years; age range, 15-79 years) were included in this study.

\section{MR Imaging Protocol}

All patients underwent MR imaging of the spine in the axial and sagittal planes on a $1.5 \mathrm{~T}$ unit (Gyroscan Intera; Philips Healthcare, Best, the Netherlands) by using a receive-only synergy spine coil. Each 1.5T study consisted of axial and sagittal T1-weighted (TR/TE, 500/10 ms) and T2-weighted (TR/TE, 4200/100 ms) images with an FOV of $160 \times 160 \mathrm{~mm}$ for the axial and $320 \times 320$ $\mathrm{mm}$ for the sagittal images. Section thickness was $4 \mathrm{~mm}$ with a $10 \%$ intersection gap on all sequences. The echo-train lengths were 3-5 for the T1-weighted images and 16-18 for the T2weighted images. A $224 \times 175$ matrix was used for the axial images; and $464 \times 232$, for the sagittal images. Of the 117 patients, 12 also underwent spine MR imaging at a local hospital.

\section{Operative Findings}

The disk surgeries were performed by 1 of 2 orthopedic surgeons. They made note of the exact location, type, and extent of each lumbar HIVD. The mean delay between MR imaging and disk surgery was 7 days (range, 0-29 days). In the operation, subligamentous HIVD was defined if the intact PLL covered the herniated disk material. Extraligamentous HIVD was defined if some portion of the herniated disk material was located posterior to the PLL and exposed to epidural space through the tear of the PLL. ${ }^{7}$

\section{MR Imaging Analysis}

Some possible MRimaging criteria were determined by a musculoskeletal radiologist with 10 years' experience. These were as follows: 1) the presence or absence of disk migration, 2) a budding appearance of the HIVD, 3) spinal canal compromised for more than half its dimension, 4) an internal signal difference in the HIVD, 5) the margin of the HIVD, 6) the integrity of the continuous low-signal-intensity line covering the HIVD, 7) the presence of an internal dark line in the HIVD, 8) adjacent BM corner erosion, 9) BM corner edema, and 10) the degree of height loss of the original disk.

The first criterion, disk migration, was interpreted as none, less than half of vertebral height, or more than half of vertebral height. The second criterion was the presence of a wasted appearance of the HIVD on the sagittal image (Fig 1). The third criterion, degree of spinal canal compromise, was determined on the axial section at the most severely compromised level as either more than or less than half of the dimension of the spinal canal. ${ }^{7}$ The fourth criterion, internal signal difference in HIVD, was noted on the T2-weighted images. An internal signal difference in HIVD was defined as a mixed low and high signal intensity inside the herniated disk material on the T2-weighted image. The fifth criterion, margin of HIVD, was interpreted as well-defined or illdefined. The sixth criterion, integrity of the continuous low-signal-intensity line covering the HIVD, was interpreted as intact, indeterminate, or interrupted on T1-weighted images (Figs 2 and 3). The seventh criterion, internal dark line in the HIVD, was

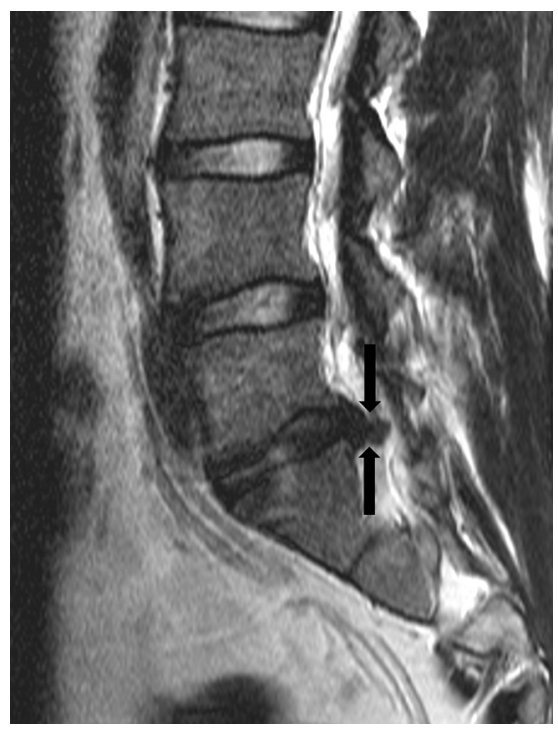

FIG 1. A 22-year-old man with HIVD at L5-S1. On T2-weighted sagittal MR imaging (TR/TE, 4200/100), the herniated disk is wasted by the PLL and it resembles budding (arrows).

interpreted as nonvisualized, indeterminate, or definitely visualized (Fig 4). The internal dark line was defined as a line with very low signal intensity on T1- and T2-weighted images traversing the herniated disk. The eighth criterion was the presence or absence of the BM corner erosion adjacent to the HIVD. The ninth criterion was the presence or absence of the BM corner edema adjacent to the HIVD. The tenth criterion, degree of height loss of original disk, was compared with adjacent normal lumbar disk height. Severe height loss was interpreted if disk height loss was more than half.

Two musculoskeletal radiologists (with 10 and 5 years' experience) retrospectively reviewed the MR images in consensus without knowledge of the clinical data or surgical findings. They focused on the above-described 10 criteria for PLL integrity. All MR images were retrospectively evaluated in random order and compared with the operative findings.

\section{Statistical Analysis}

Sensitivity, specificity, accuracy, PPV, NPV, and odds ratio of each MR imaging criterion for extraligamentous HIVD were calculated by univariate analysis. The predictive values of the MR imaging criteria were calculated by using the Pearson $\chi^{2}$ test. Differences with a $P$ value $<.05$ were considered statistically significant. Inter- and intraobserver agreement was calculated by using $\kappa$ coefficients.

\section{RESULTS}

Of the 117 patients, 5 underwent open diskectomy and 112 underwent microdiskectomy. There were 66 (56\%) cases of extraligamentous lumbar HIVD and 51 (44\%) cases of subligamentous lumbar HIVD. The symptomatic lumbar disk herniation levels were L2-3 in 1 patient, L3-4 in 12 patients, L4-5 in 59 patients, and L5-S1 in 45 patients.

The sensitivity, specificity, accuracy, and $P$ value of each of the $10 \mathrm{MR}$ imaging criteria are shown in Table 1. Among the 10 MR imaging criteria, the following 5 are suggestive of 

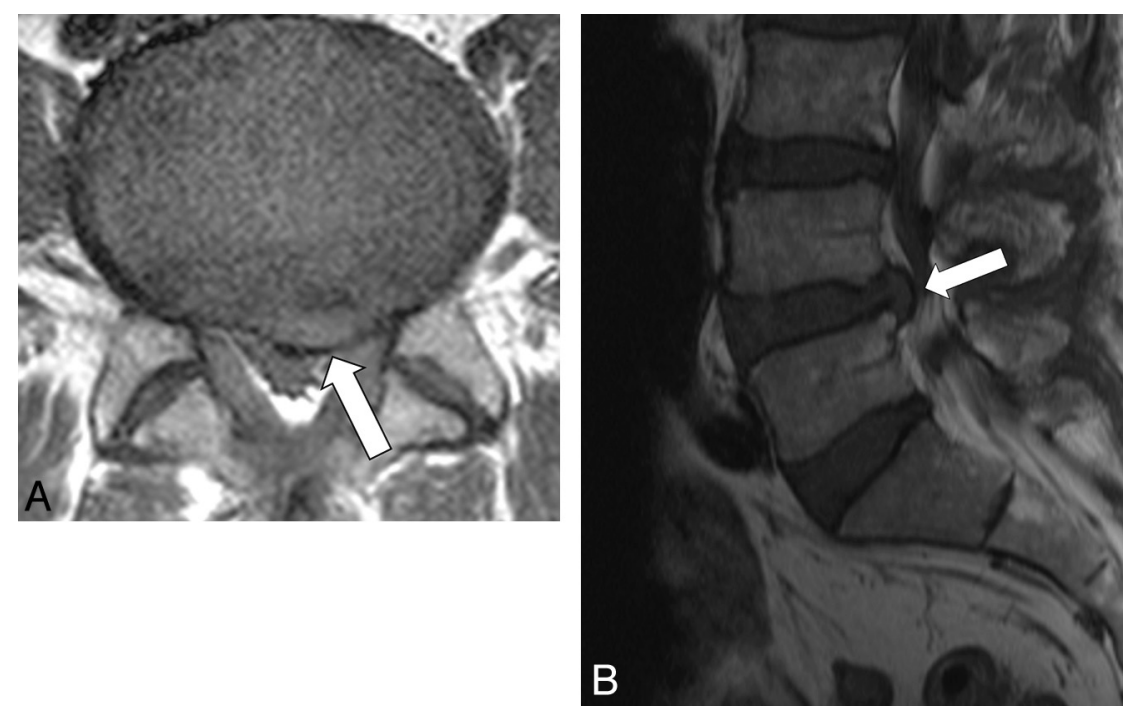

FIG 2. A 65-year-old man with a subligamentous HIVD at L4-5. T7-weighted axial (A) and T7weighted sagittal $(B)$ MR images (TR/TE, 500/10 ms) show the intact low-signal-intensity line covering the HIVD.
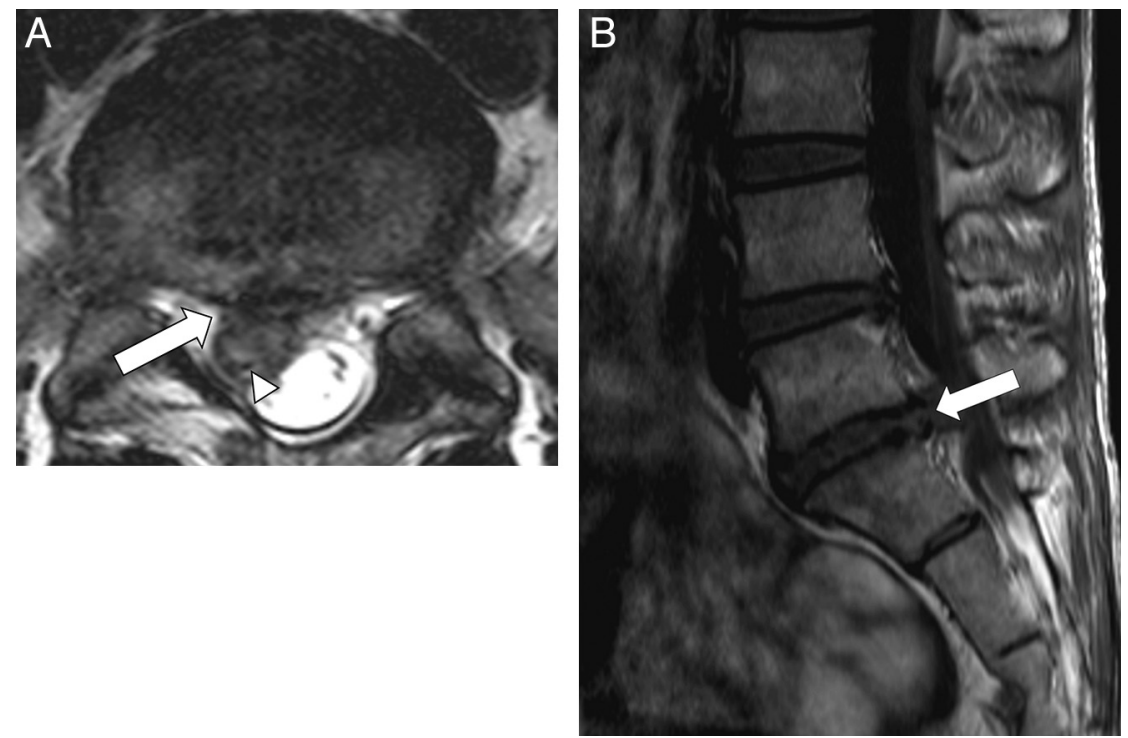

FIG 3. A 36-year-old man with an extraligamentous HIVD at L5-S1. A, T2-weighted axial MR image (TR/TE, 4200/100 ms) shows disruption of the continuous low-signal-intensity line covering the herniated disk (arrow) and the internal dark line (arrowhead). B, T1-weighted sagittal MR image (TR/TE, 500/10 ms) shows disruption of the continuous low-signal-intensity line covering the herniated disk (arrow).

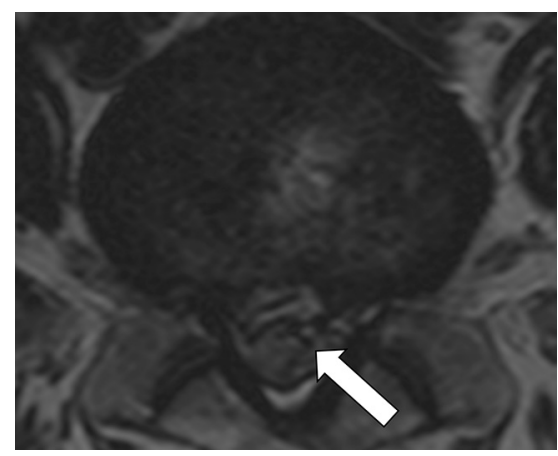

FIG 4. A 47-year-old man with extraligamentous HIVD at L5-S1. On the T2-weighted axial MR image (TR/TE, 4200/100 ms), the internal dark line (arrow) is interposed in the herniated disk. extraligamentous HIVD rather than subligamentous $\operatorname{HIVD}(P<.05): 1)$ spinal canal compromised more than half its dimension, 2) internal signal difference in the HIVD, 3) margin of the HIVD, 4) disruption of the continuous low-signal-intensity line covering the HIVD, and 5) the presence of an internal dark line in the HIVD.

Comparing the criteria, we found that the $66.7 \%$ accuracy of the disruption of the continuous low-signal-intensity line covering the HIVD was better than the $61.5 \%$ accuracy of the spinal canal compromise, the $56.4 \%$ accuracy of the presence of an internal dark line in the HIVD, the $55.6 \%$ accuracy of internal signal difference in the HIVD, and the $50.4 \%$ accuracy of the margin of the HIVD. When we combine all $5 \mathrm{MR}$ imaging criteria, the accuracy $(76.1 \%)$ was improved.

Statistically, 5 significant MR imaging criteria and their combined sensitivity, specificity, accuracy, PPV, NPV, and odds ratio are shown in Table 2. The odds ratio for the combination of the $5 \mathrm{MR}$ imaging criteria was $9.93(P<.0001)$.

The intra- and interobserver agreement was almost perfect: respectively, $\kappa$ value, 0.93 and 0.91 for the presence of disk migration; 0.95 and 0.93 for the budding appearance in the HIVD; 0.92 and 0.91 for the spinal canal compromised for more than half its dimension; 0.83 and 0.81 for the internal signal difference in the HIVD; 0.87 and 0.84 for the ill-defined margin of the HIVD; 0.85 and 0.83 for the disruption of the continuous low-signalintensity line covering the HIVD; 0.84 and 0.81 for the presence of an internal dark line in the HIVD; 0.93 and 0.91 for the adjacent bone marrow corner erosion; 0.93 and 0.91 for the bone marrow corner edema; and 0.97 and 0.95 for the severe height loss of the original disk.

\section{DISCUSSION}

PLL has a denticulated configuration, with a narrow retrovertebral segment and a wide retrodiskal segment. PLL of the lumbar spine covers the outer fiber of the annulus fibrosus of the disk. At the level of the disk, PLL adheres to the posterior aspect of the disk and extends laterally beyond the foramen. ${ }^{5}$ PLL is seen as a very low-signal-intensity line with 1 layer on all MR imaging pulse sequences. When we measured its thickness at the L5-S1 intervertebral disk level on T1-weighted sagittal images, its mean thickness was approximately $9.7 \mathrm{~mm}$ (range, $5.4-11.5 \mathrm{~mm}$ ). The interface between the PLL and the outer fibers of annulus is some- 
Table 1: Evaluation of MR imaging criteria for indicating extraligamentous HIVD in the lumbar spine

\begin{tabular}{|c|c|c|c|c|}
\hline MR Imaging Criteria & Sensitivity (\%) & Specificity (\%) & Accuracy (\%) & $P$ Value \\
\hline Presence of disk migration & $50 / 66(75.8)$ & $20 / 51(39.2)$ & $70 / 117(59.8)$ & .0817 \\
\hline Budding appearance in HIVD & $15 / 66(22.7)$ & $43 / 51(84.3)$ & $58 / 117(49.6)$ & .3427 \\
\hline Spinal canal compromised for more than half its dimension & $30 / 66(45.5)$ & $42 / 51(82.4)$ & $72 / 117(61.5)$ & .0015 \\
\hline Internal signal difference in HIVD & $23 / 66(34.8)$ & $42 / 51(82.4)$ & $65 / 117(55.6)$ & .0385 \\
\hline Ill-defined margin of HIVD & $10 / 66(15.2)$ & $49 / 51(96.1)$ & $59 / 117(50.4)$ & .0471 \\
\hline Disruption of continuous low-signal-intensity line covering HIVD & $38 / 66(57.6)$ & $40 / 51(78.4)$ & $78 / 117(66.7)$ & $<.0001$ \\
\hline Presence of internal dark line in HIVD & $20 / 66(30.3)$ & $46 / 51(90.2)$ & $66 / 117(56.4)$ & .0072 \\
\hline Adjacent bone marrow corner erosion & $35 / 66(53.0)$ & $29 / 51(56.9)$ & $64 / 117(48.6)$ & .2877 \\
\hline Bone marrow corner edema & $19 / 66(28.8)$ & $42 / 51(82.4)$ & $61 / 117(52.1)$ & .1615 \\
\hline Severe height loss of original disk & $61 / 66(92.4)$ & $6 / 51(11.8)$ & $67 / 117(57.3)$ & .3238 \\
\hline
\end{tabular}

Table 2: MR imaging criteria suggestive of extraligamentous HIVD in the lumbar spine

\begin{tabular}{lcccccc}
\hline \multicolumn{1}{c}{ MR Imaging Criteria } & Sensitivity (\%) & Specificity (\%) & Accuracy (\%) & PPV (\%) & NPV (\%) & Odds Ratio/P Value \\
\hline $\begin{array}{l}\text { Spinal canal compromised for more } \\
\text { than half its dimension }\end{array}$ & $30 / 66(45.5)$ & $42 / 51(82.4)$ & $72 / 117(61.5)$ & $30 / 39(76.9)$ & $42 / 78(53.8)$ & $3.88 / .0015$ \\
Internal signal difference in HIVD & $23 / 66(34.8)$ & $42 / 51(82.4)$ & $65 / 117(55.6)$ & $23 / 32(71.9)$ & $42 / 85(49.4)$ & $2.49 / .0385$ \\
IIl-defined margin of HIVD & $10 / 66(15.2)$ & $49 / 51(96.1)$ & $59 / 117(50.4)$ & $10 / 12(83.3)$ & $49 / 105(46.7)$ & $4.37 / .0471$ \\
$\begin{array}{l}\text { Disruption of continuous low-signal- } \\
\text { intensity line covering HIVD }\end{array}$ & $38 / 66(57.6)$ & $40 / 51(78.4)$ & $78 / 117(66.7)$ & $38 / 49(77.6)$ & $40 / 68(58.8)$ & $4.93 /<.0001$ \\
$\begin{array}{l}\text { Presence of internal dark line in HIVD } \\
\text { Combination of 5 MR imaging criteria }\end{array}$ & $20 / 66(30.3)$ & $46 / 51(90.2)$ & $66 / 117(56.4)$ & $20 / 25(80.0)$ & $46 / 92(50.0)$ & $4.00 / .0072$ \\
\hline
\end{tabular}

times indistinguishable. ${ }^{5}$ In evaluating the PLL, the T1-weighted images were more accurate than the proton attenuationweighted and T2-weighted images. ${ }^{5}$

There are only 2 reports of PLL integrity in the lumbar HIVD on MR images. ${ }^{5,6}$ Grenier et $a l,{ }^{5}$ in a prospective study of 17 patients with 19 lumbar disk herniations, reported 100\% sensitivity and $78 \%$ specificity of MR imaging in detecting PLL disruption. On the other hand, Silverman et $\mathrm{al}^{6}{ }^{6}$ in a prospective study of 50 patients with 33 subligamentous and 17 supraligamentous HIVDs, reported 29\% sensitivity, 65\% specificity, and $42 \%$ accuracy of MR imaging in the presence of the continuous low-signalintensity line posterior to the disk herniation. In our study, disruption of the continuous low-signal-intensity line covering the HIVD had a $57.6 \%$ sensitivity, $78.4 \%$ specificity, and $66.7 \%$ accuracy.

We thought that when the interrupted PLL was interposed in the HIVD, we would see the internal dark line. Therefore, its signal intensity was compared with adjacent normal PLL signal intensity. The results of our study showed that the presence of an internal dark line in a herniated disk was a reliable marker for extraligamentous HIVD. We found the internal dark line in 20 (30\%) of 66 patients with extraligamentous HIVD and 5 (10\%) of 51 with subligamentous HIVD.

If the disk extruded through the PLL (transligamentous HIVD), the HIVD would have a budding appearance because the herniated disk could be wasted by the PLL. In the beginning of this study, we thought that a budding appearance would be a good sign of a transligamentous HIVD. However, this finding was not specific for extraligamentous HIVD, according to our study. We thought the budding appearance could also be seen in a subligamentous HIVD if the HIVD was wasted by either a deep layer of the PLL with an intact superficial layer of the PLL or an outer annulus in an already-bulged disk.
In this study, we found 5 helpful MR image criteria to suggest extraligamentous HIVD: 1) spinal canal compromised for more than half its dimension, 2) an internal signal difference in the HIVD, 3) an ill-defined margin of the HIVD, 4) disruption of the continuous low-signal-intensity line covering the HIVD, and 5) the presence of an internal dark line in the HIVD (Table 1). When we combined these $5 \mathrm{MR}$ imaging criteria, the sensitivity (77.3\%), specificity $(74.5 \%)$, and accuracy $(76.1 \%)$ were improved but the improvement was moderate at best. These results are probably due to the similar features of each of the $5 \mathrm{MR}$ imaging criteria, so noticeable increases of the sensitivity, specificity, and accuracy of the combined $5 \mathrm{MR}$ imaging criteria were difficult to perceive.

There are some limitations to our study. First, this is a retrospective study, so some cases were excluded due to an incomplete description of the PLL integrity on the surgical record. Second, the PLL integrity was determined by the surgeon's observation only, so small defects in the PLL and attenuation of part or all of the PLL might not have been detected. Third, the MR imaging interpretation was made by radiologists in the same hospital. Fourth, logistic regression analysis was done because of the multicolinearity effect.

\section{CONCLUSIONS}

Our proposed $5 \mathrm{MR}$ imaging criteria will be helpful in differentiating extraligamentous and subligamentous HIVD in the lumbar spine.

Disclosures: Jin S. Yeom-UNRELATED: Payment for Lectures (including service on Speakers Bureaus): Medtronic (\$5000).

\section{REFERENCES}

1. Onik G, Helms CA, Ginsburg L, et al. Percutaneous lumbar diskectomy using a new aspiration probe. AJR Am J Roentgenol 1985;144: $1137-40$ 
2. Kambin P, Sampson S. Posterolateral suction-excision of herniated lumbar intervertebral discs: report of interim results. Clin Orthop 1986;207:37-43

3. Onik G, Helms CA. Automated percutaneous lumbar discectomy. AJR Am J Roentgenol 1991;156:531-38

4. Mink JH. Imaging evaluation of a candidate for percutaneous lumbar discectomy. Clin Orthop 1989;238:83-91

5. Grenier N, Greselle J, Vital J, et al. Normal and disrupted lumbar longitudinal ligaments: correlative MR and anatomic study. Radiology 1989;171:197-205
6. Silverman CS, Lenchik L, Shimkin PM, et al. The value of MR in differentiating subligamentous from supraligamentous lumbar disk herniations. AJNR Am J Neuroradiol 1995;16:571-79

7. Fardon D, Milette P, for the Combined Task Forces of the North American Spine Society, American Society of Spine Radiology, and American Society of Neuroradiology. Nomenclature and classification of lumbar disc pathology: recommendations of the combined task forces of the North American Spine Society, American Society of Spine Radiology, and American Society of Neuroradiology. Spine (Phila Pa 1976) 2001;26:E93-113 